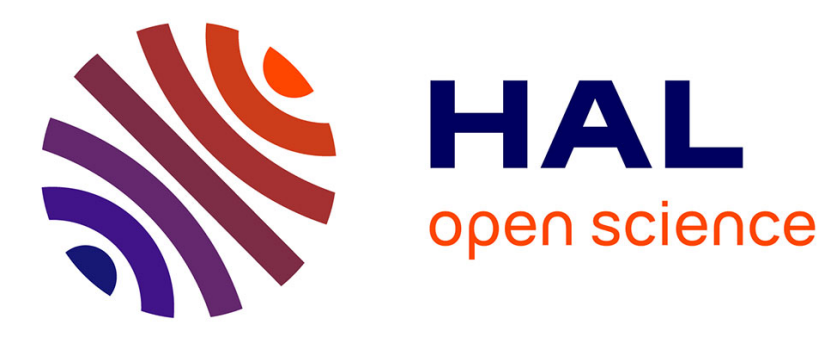

\title{
Underwater Color Constancy: Enhancement of Automatic Live Fish Recognition
}

M. Chambah, D. Semani, Arnaud Renouf, P. Coutellemont, A. Rizzi

\section{To cite this version:}

M. Chambah, D. Semani, Arnaud Renouf, P. Coutellemont, A. Rizzi. Underwater Color Constancy: Enhancement of Automatic Live Fish Recognition. 16th Annual symposium on electronic imaging, 2004, Inconnue, United States. pp.157-168. hal-00263734

\section{HAL Id: hal-00263734 https://hal.science/hal-00263734}

Submitted on 13 Mar 2008

HAL is a multi-disciplinary open access archive for the deposit and dissemination of scientific research documents, whether they are published or not. The documents may come from teaching and research institutions in France or abroad, or from public or private research centers.
L'archive ouverte pluridisciplinaire HAL, est destinée au dépôt et à la diffusion de documents scientifiques de niveau recherche, publiés ou non, émanant des établissements d'enseignement et de recherche français ou étrangers, des laboratoires publics ou privés. 


\title{
Underwater Color Constancy : Enhancement of Automatic Live Fish Recognition
}

\author{
M. Chambah, D. Semani, A. Renouf, P. Courtellemont, A. Rizzi* \\ L3I, Université de La Rochelle, France \\ E-mail : \{mchambah, dsemani, arenouf, pcourtel $\} @$ univ-lr.fr \\ *Dept. of Information Technology - University of Milano/Italy \\ E-mail: rizzi@dti.unimi.it
}

\begin{abstract}
We present in this paper some advances in color restoration of underwater images, especially with regard to the strong and non uniform color cast which is typical of underwater images. The proposed color correction method is based on ACE model, an unsupervised color equalization algorithm. ACE is a perceptual approach inspired by some adaptation mechanisms of the human visual system, in particular lightness constancy and color constancy. A perceptual approach presents a lot of advantages: it is unsupervised, robust and has local filtering properties, that lead to more effective results. The restored images give better results when displayed or processed (fish segmentation and feature extraction). The presented preliminary results are satisfying and promising.
\end{abstract}

Keywords: Automatic fish species recognition, underwater imaging, color constancy, automatic color correction, color pattern recognition, color features.

\section{THE AQU@THEQUE PROJECT}

The Aqu@ $@$ thèque project consists in developing an information system dedicated to aquariums. The fish tanks are filmed in live by a remote video camera. The system allows an aquarium visitor to remote control the camera and select a fish of his choice on an interactive interface by pointing out the fish on a touch screen.

Then the selected fish is displayed on the screen and automatically identified using a real-time recognition method. Educational information and a virtual representation of the identified fish in its natural environment are also displayed in real time on another screen.

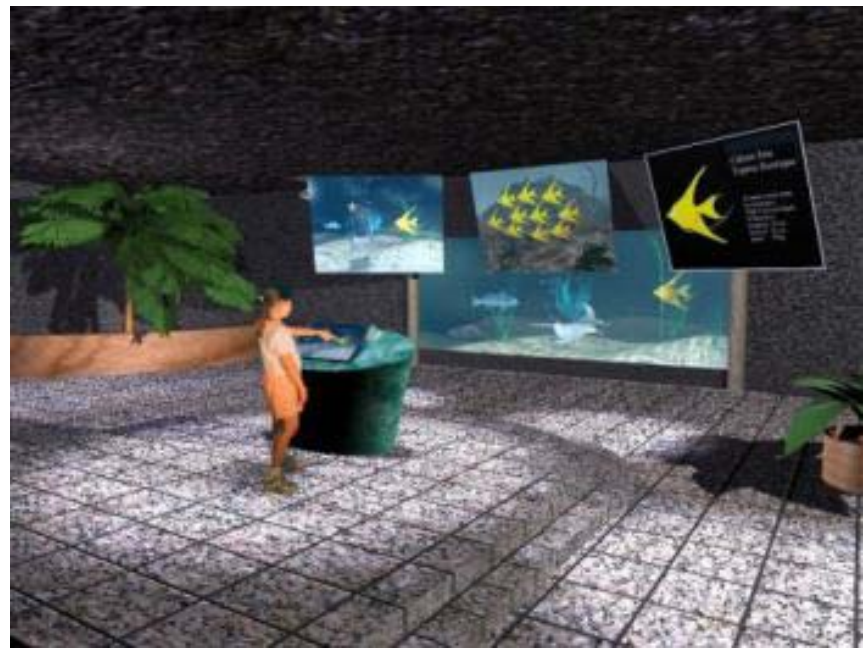

Fig. 1 : The aqu@ thèque interactive environment 
The project is based on an automatic recognition method, an educational information retrieval system and a behavioral modeling system of virtual fish. In this paper, we focus on the real-time recognition part of the system and especially on color correction and color cast removal from the videos due to the aquatic environment.

The recognition system consists in three steps:

- A segmentation step that extracts the main regions corresponding to fishes in video sequences.

- A feature extraction step based on the segmentation results.

- A fish classification step among the different species present in the tank.

The works that preceded this article focused, on one hand, on the segmentation process, feature extraction step and the classification step [1][2][7]. On the other hand, an algorithm for digital images unsupervised enhancement, called ACE for Automatic Color Equalization was also presented [4][5][10]. It provided experimental evidence for correcting automatically the color balance of an image using a perceptual approach.

We present in this paper the preliminary results of a new technique for underwater images color restoration based on ACE. This work is a collaboration between the L3I lab of Université de La Rochelle and the Department of Information Technology of University of Milano.

After comparing the results of some usual color constancy methods with the results of our proposed technique, we present the impact of the color correction on the next steps i.e. the segmentation and the fish classification step. We report and discuss the results of our experiments and we give some future prospects of this work.

\section{UNDERWATER COLOR CONSTANCY}

The videos taken in aquatic environment present a strong and non uniform color cast. It means that the cast has different color and intensity in foreground, background (more depth), shadows and highlights. This cast has to be removed.

The images taken from the tanks of the aquarium can be mainly of three kinds. The first one is an image with an overall view with different species of fish. This kind of images is the most chromatically diverse (see fig. 3). The second kind consists in an image with some fish belonging to the same species (see fig. 15). The third kind consists in image with a close view of one fish (see fig. 19). The latter type of images is the less chromatically diverse and hence the hardest to balance.

Since there is no universal color constancy method, we retain, for the experiments, the non-use of a priori information since the non-supervision is an important factor in live videos, and the applicability of the method on natural scenes, as selection criteria among the several existing methods of color constancy.

Among the methods responding to these criteria there are gray world (GW) and Retinex white patch (WP), which are widely used and often give good results. These methods however, are designed to remove the color cast caused by an illuminant shift, while we want to correct the cast due to an aquatic environment plus an artificial illumination in this environment. Other retained methods are the GW/WP hybrid method [3] and the ACE method [4][5][10].

The GW/WP hybrid method is designed to handle more than a cast in the same image. It consists in a progressive combination of GW and WP to estimate and correct color cast in highlights, mid-tones and shadows.

The ACE method, for Automatic Color Equalization, is an algorithm for digital images unsupervised enhancement. It is based on a new computational approach that merges the Gray World and White Patch equalization mechanisms, while taking into account the spatial distribution of color information. It is inspired by some adaptation mechanisms of the human visual system (HVS), in particular lightness constancy and color constancy. Lightness constancy makes us stably perceive the scene regardless changes in mean luminance intensity and color constancy makes us stably perceive the scene regardless changes in color of the illuminant.

ACE is able to adapt to widely varying lighting conditions, and to extract visual information from the environment efficaciously. 


\subsection{Experimental results}

For these experiments we test each color constancy method on each of the three kinds of image described above (from overview to close-up), varying in this way the chromatic diversity of the images.

The image of fig. 3.a represents different fish species in an overall view. It has different casts (depending on the water depth and the illuminant) but especially a strong cyan-green cast as shown by the hue histogram of fig. 3.b.

The RGB histogram of the image (fig. 9) has a poor dynamic range and clipped values, hence the necessity of enhancement of the image.

The GW method gives an overall color balanced image, since the original image is chromatically diverse (fig. 4.a), but the obtained image is not totally chromatically diverse, there is a slight reddish cast (fig. 4.b), the background fish have some cast left and the image has a poor contrast and is oversaturated (fig. 10).

Since the original image is oversaturated (some clipped values at the end of the histogram), the WP method does not change much the original one (fig. 6 and 12).

The GW/WP hybrid method gives a result similar to GW (fig. 5 and 11), since WP has no effect on the image.

Inner parameters of the ACE algorithm were properly tuned to meet the requirements of image and histogram shape naturalness and to deal with this kind of aquatic images. We chose 0.2 for saturation parameter as a trade off between a good Color Constancy behavior and a solution that does not amplify excessively the original low contrast background noise. We experimented the algorithm with the "keep original gray" (fade to black) feature switched on and off. The "keep original gray" feature has been devised to relax the GW mechanism in the second stage: instead of centering the chromatic channels around the medium gray, "keep original gray" preserve the original mean values; this results in histograms more similar in shape with original ones.

With the "keep original gray" feature enabled, the background cast still remains (fig. 7) due to the strong original color cast. But the RGB histogram (fig. 13) of the image has a good dynamic and a natural shape with no effects of equalization.

The best result is obtained when the "keep original gray" feature is disabled. In fact, all the casts are removed from the image (fig. 8.a), the image is chromatically diverse (fig. 8.b) and the histogram shows a good dynamic and a natural shape (see fig. 14).

The image of fig. 15.a represents some fish belonging to the same species. It has a strong green-cyan cast as shown by the hue histogram on fig. 15.b.

The GW method estimates the most prominent cast in the image, since it is based on the mean of the image. As a consequence, it corrects the mid-tones and the shadows of the image, i.e. especially the background of the image, as illustrated by fig. 16.a, inverting the color cast in the foreground, hence the magenta cast (fig. 16.b) present in the foreground of the image.

Since the WP has no effect on the image, the GW/WP hybrid method gives similar results to GW with less reverse magenta cast (fig. 17.a).

The ACE method (without "keep original gray" feature) gives a correct color of fish (fig. 18.a), a good chromatic diversity (fig. 18.b) and much less reverse cast than the other methods.

The image of fig. 19.a represents a close-up of one fish. It has a poor chromatic diversity and it is hence hard to correct. As for fig. 15.a, GW corrects the most prominent cast in the image and inverts the cast in the foreground (fig. 20). Since the WP has nearly no effect on the image (fig. 22), the GW/WP hybrid method gives similar results to GW with less reverse magenta cast (fig. 21.a). The ACE method (without "keep original gray" feature) gives the best result with a correct color of fish (fig. 23.a), a good chromatic diversity (fig. 23.b) and much less reverse cast than the other methods. 
As a conclusion, usual color constancy methods such as gray world and white patch are not efficient for such kind of images, since they are global and thus able to handle only uniform color casts. The GW method estimates the most prominent cast in the image, since it is based on the mean of the image. As a consequence, it corrects the mid-tones and the shadows of the image, inverting the color cast in the foreground. The white patch method corrects the highlights. This result is logical since this method estimates the cast in highlights. The shadows and mid-tones still suffer from a cast since the cast is non uniform. Moreover, WP is very sensitive to noise and to clipping. The videos of the fish tanks can be over saturated (so some are clipped), depending of the variable lighting conditions, so this method is not suitable to underwater videos color correction.

ACE gives the best results since it widely adapts to different non uniform color casts and it is unsupervised.

\section{IMPACT ON THE FISH SEGMENTATION STEP}

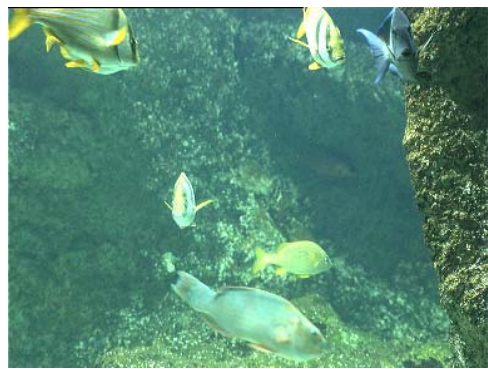

Original image

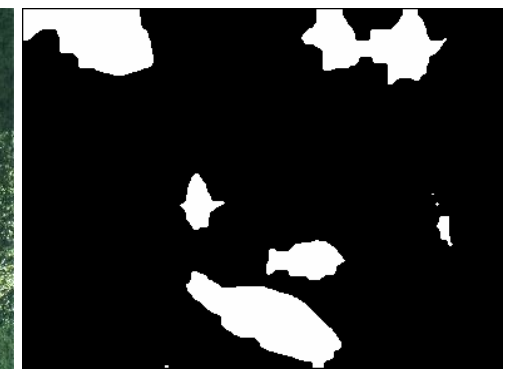

Segmented image

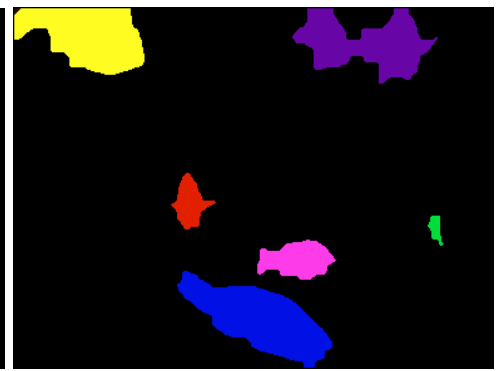

Labeled image

Fig. 2 : The segmentation step

The segmentation step [1-2] is a decisive step for the correct recognition of fish. It consists in a background subtraction. Typically, a video sequence includes a static background and moving fish translating and warping along consecutive frames. This phase is to breaks up an image into regions of interest representing the fish.

We compared the segmentation results on original videos and on corrected videos with the proposed method (ACE without "keep original gray" feature). The fish are better localized on the corrected videos than on the original ones as shown by the figs 24 and 25 . Some very small false detections are noticed, but they can easily discarded using a threshold.

The color cast removal enhances the fish segmentation step, since it emphasizes the color differences between the fish and the background. Moreover, the color features extracted from the balanced images are closer to the real colors of the fish [5].

The segmentation of an aquatic environment is a very difficult issue due to the variability of illumination and to the consequent color cast. In the segmentation and the color features extraction steps, assumptions could be made concerning the constancy of the illuminant and the color cast. These assumptions may be true as long as the recognition and the learning take place in the same fish tank and from the same position, but they are no longer true if the learning and the recognition are done on different fish tanks or from different positions. Hence the importance of a color balancing and color cast removal from the live video sequences before the fish recognition step performed without any a priori information or assumption about the scene.

\section{IMPACT ON THE FISH RECOGNITION STEP}

Among the features extracted (during the second step) for the classification and recognition of fishes there are geometric features (e.g. area, perimeter, roundness ratio, elongation, orientation), color features (e.g. hue, gray levels, color histograms, chrominance values), texture features (e.g. entropy, correlation) and motion features. We integrated also some new color features, such as generalized color moments and color correlograms [8-9].

In order to eliminate not useful or redundant features, a feature selection step based on an ambiguity measure is applied to select the most pertinent ones [7]. Feature reduction aims to make the classification process easier and to speed up the recognition step up in order to achieve a real-time processing. Finally, the quadratic Bayes classifier is used to classify the selected fishes to one of the learned species. 
We compared the recognition results between the original images and the corrected images using 85 extracted features, 12 fish species and 1346 regions (representing fish). The preliminary results give nearly the same error rate for the original images and the corrected ones $(14.23 \% \pm 0.17)$ vs. $(14.87 \% \pm 0.27)$. The reported error rates are the mean of the ten-fold cross-validation.

The centering and reduction of the extracted features (done to avoid the impact of the color cast) is one of the reasons for the similarity of the error rate. Better color features should be extracted to take advantage of the removal of the color cats in the corrected images.

\section{CONCLUSION}

We have presented in this paper an automatic method for underwater images color cast removal. The underwater videos present often a non-uniform color cast due to the depth of the water and the artificial illumination.

The proposed ACE based method adapts to a variety of color casts and different kinds of images (overview to closeup) with no a priori knowledge about the scene.

In addition to the enhancement of the displayed video, the color cast removal allows a better fish localization and segmentation.

One of the possible future prospects of this work is to increase the recognition rate by extracting more suitable color features that describe more closely the fish.

\section{REFERENCES}

1. D.Semani, T.Bouwmans, C.Frélicot, and P. Courtellemont, «Automatic Fish Recognition in Interactive Live Video», International Workshop on IVRCIA, The 6th World Multi-Conference on SYSTEMICS, CYBERNETICS AND INFORMATICS, SCI 2002, Orlando, Florida (USA), July 14-18, 2002.

2. D.Semani, C. Saint-Jean, C. Frélicot, T. Bouwmans, and P. Courtellemont, «Alive fishes species characterization from video sequences», SSPR2002 \& SPR2002 9th International workshop on Structural and Syntactic Pattern Recognition, 4th International workshop on Statistical Techniques in Pattern Recognition, Windsor, Canada, August 6-9 2002.

3. M. Chambah, B. Besserer, P. Courtellemont, "Recent Progress in Automatic Digital Restoration of Color Motion Pictures", SPIE Electronic Imaging 2002, San Jose, CA, USA, janvier 2002, vol. 4663, pp. 98-109.

4. A. Rizzi, C. Gatta, D. Marini, "From Retinex to ACE: Issues in Digital Images Unsupervised Color Equalization", Journal of Electronic Imaging, Vol. 13, No. 1, January 2004.

5. M. Chambah, A. Rizzi, C. Gatta, B. Besserer, D. Marini, "Perceptual approach for unsupervised digital color restoration of cinematographic archives", Electronic Imaging 2003, 21-24/01/03, S. Clara, California (USA).

6. M. Chambah, B. Besserer, P. Courtellemont, "Approach to Automate Digital Restoration of Faded Color Films", IS\&T Conference on Graphics, Imaging, and Vision CGIV 2002, Poitiers, France, avril 2002, pp. 613-618.

7. D. Semani, C. Frélicot, and P. Courtellemont, "Feature selection using an ambiguity measure based on fuzzy OR-2 operators", International Fuzzy Systems Association, IFSA'2003 - Istanbul - 2003.

8. F. Mindru, T. Moons, L. Van Gool, "Color-based Moment Invariants for the Viewpoint and Illumination Independent Recognition of Planar Color Patterns”, Proc. ICAPR'98, Plymouth, UK, 1998.

9. J. Huang, S. R. Kumar, M. Mitra, W.-J. Zhu, R. Zabih, "Image indexing using color correlograms", In Proc. IEEE Comp. Soc. Conf. Comp. Vis. and Patt. Rec., pages 762--768, 1997.

10. A. Rizzi, C. Gatta, D. Marini, "A New Algorithm for Unsupervised Global and Local Color Correction", Pattern Recognition Letters, Vol 24 (11), pp. 1663-1677, July 2003. 

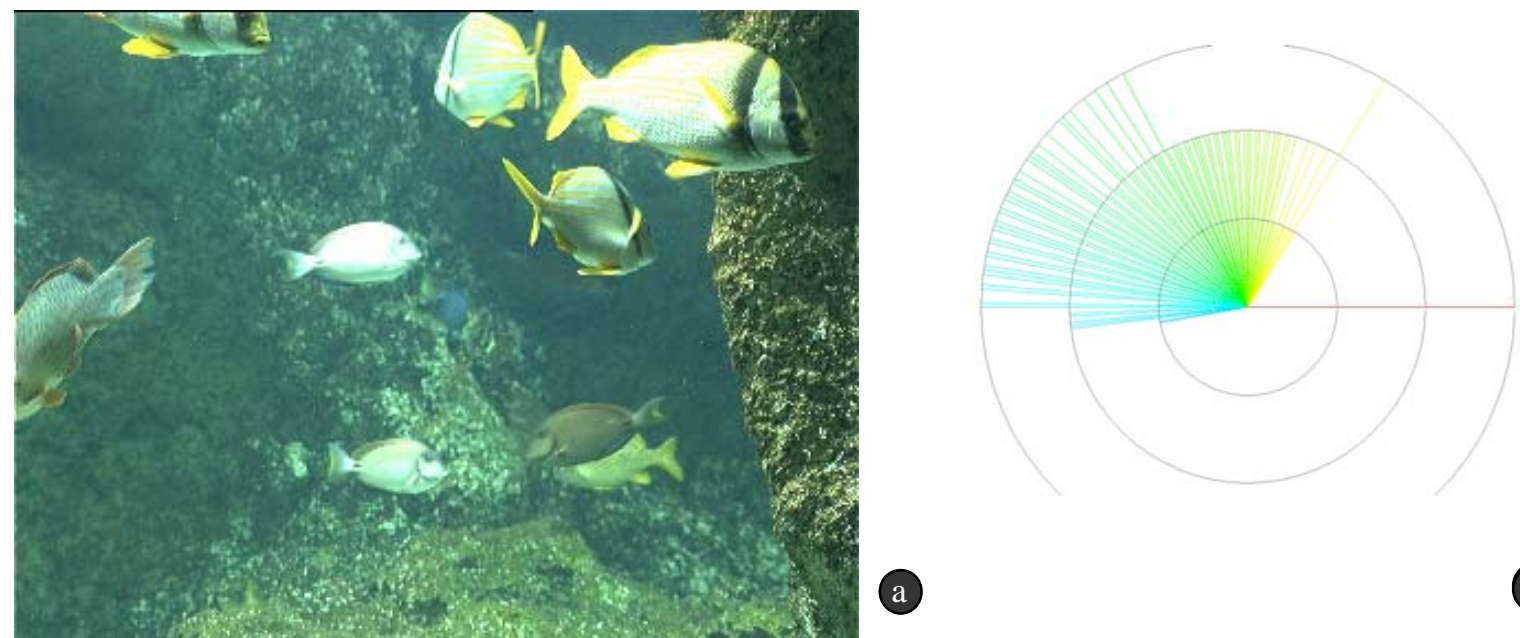

(a)

(b)

Fig.3 : Original image : overview with different fish species (a) and its polar hue histogram (b).
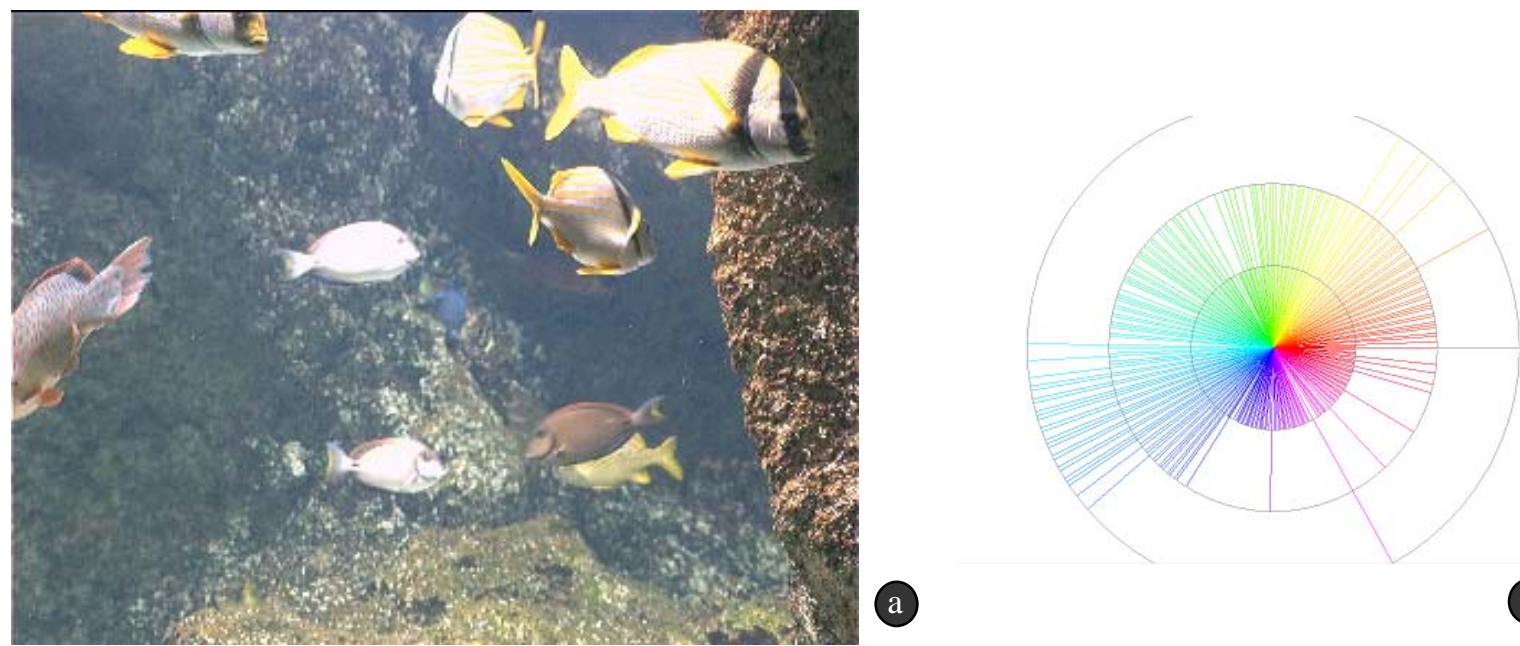

Fig. 4 : Image of fig. 3 after GW, only the predominant cast is removed (a) and its hue histogram (b).
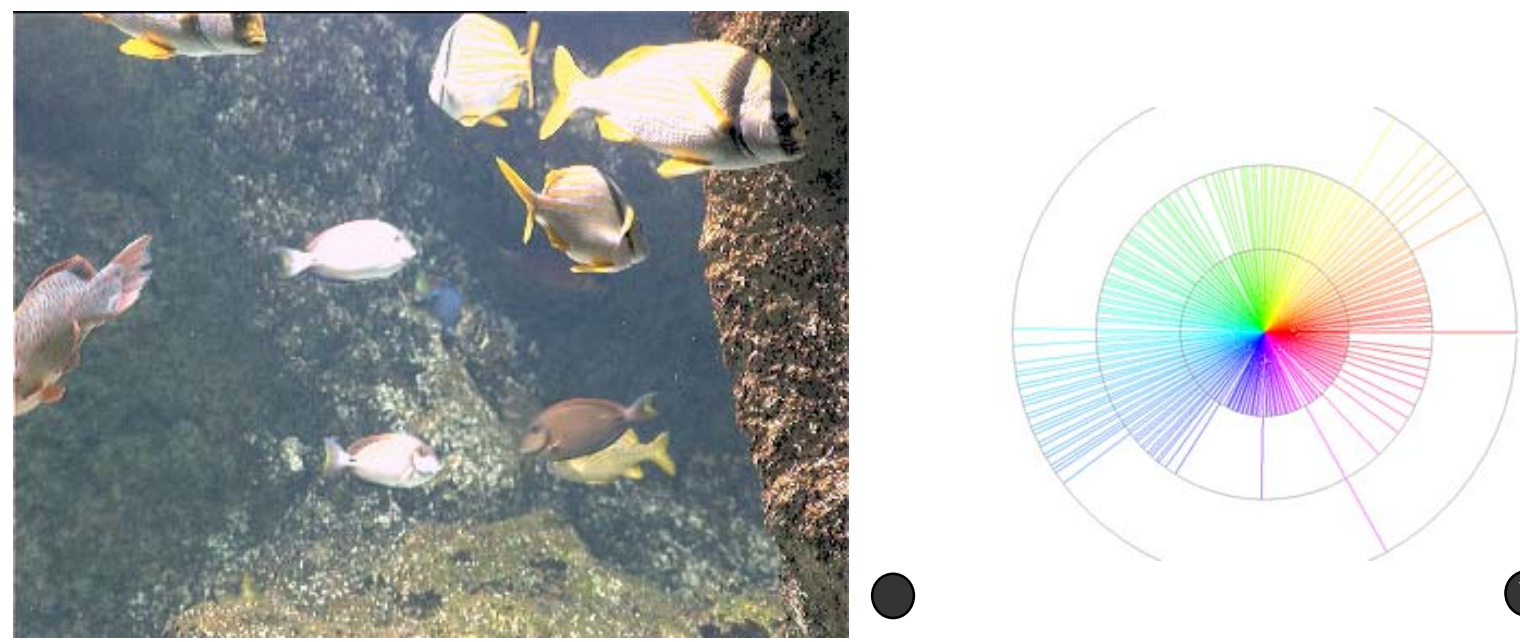

Fig. 5: Image of fig. 3 after GW/WP hybrid method (a) and its hue histogram (b). 

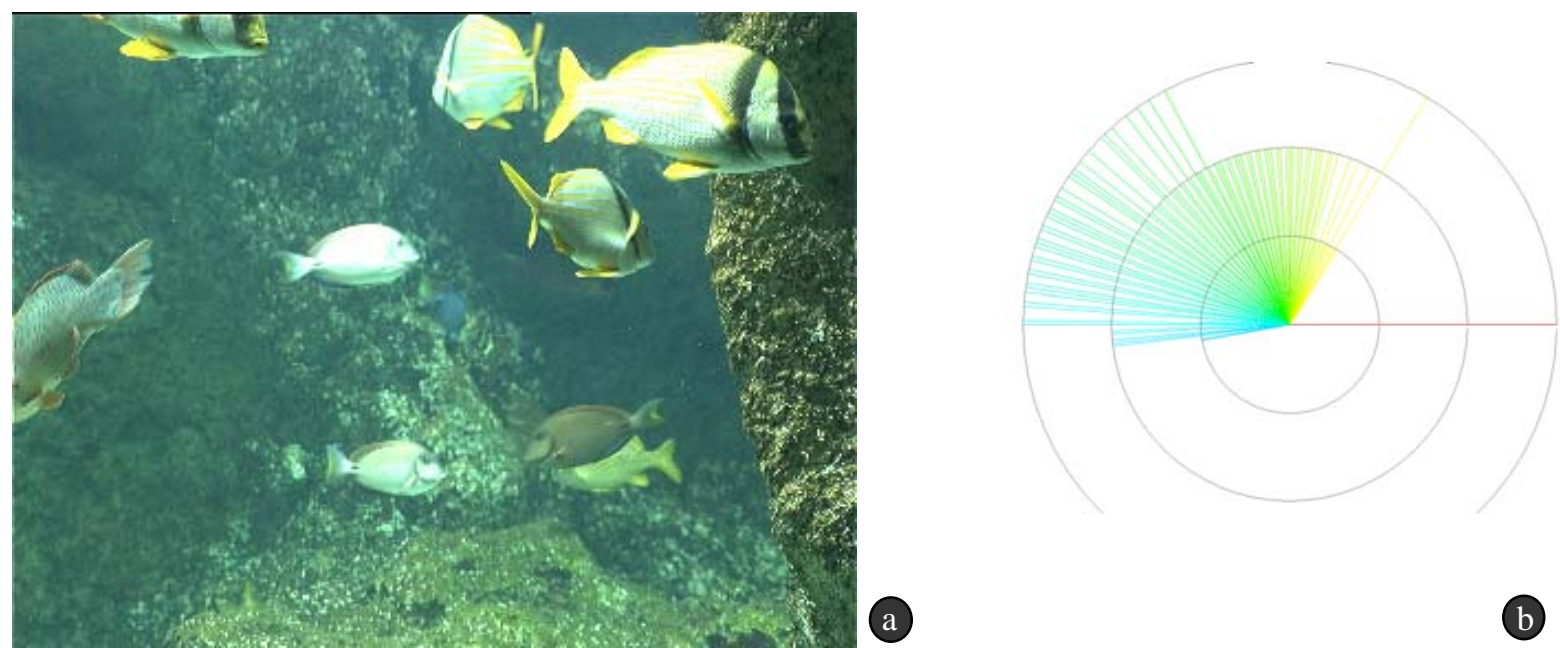

Fig.6 : Image of fig. 3 after WP (a). No result is noticed neither on the image nor on the hue histogram (b).
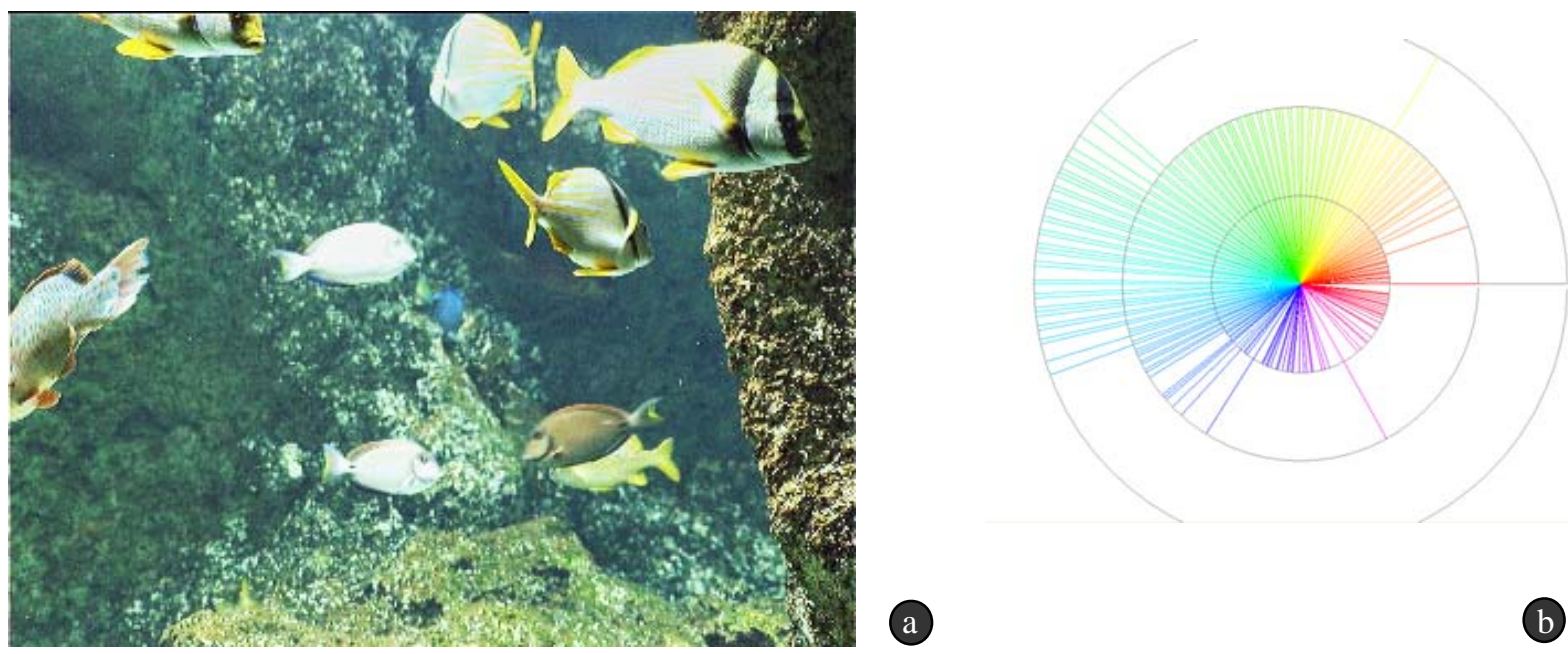

Fig. 7 : Image of fig. 3 after ACE with « keep original gay » feature (a) and its hue histogram (b).
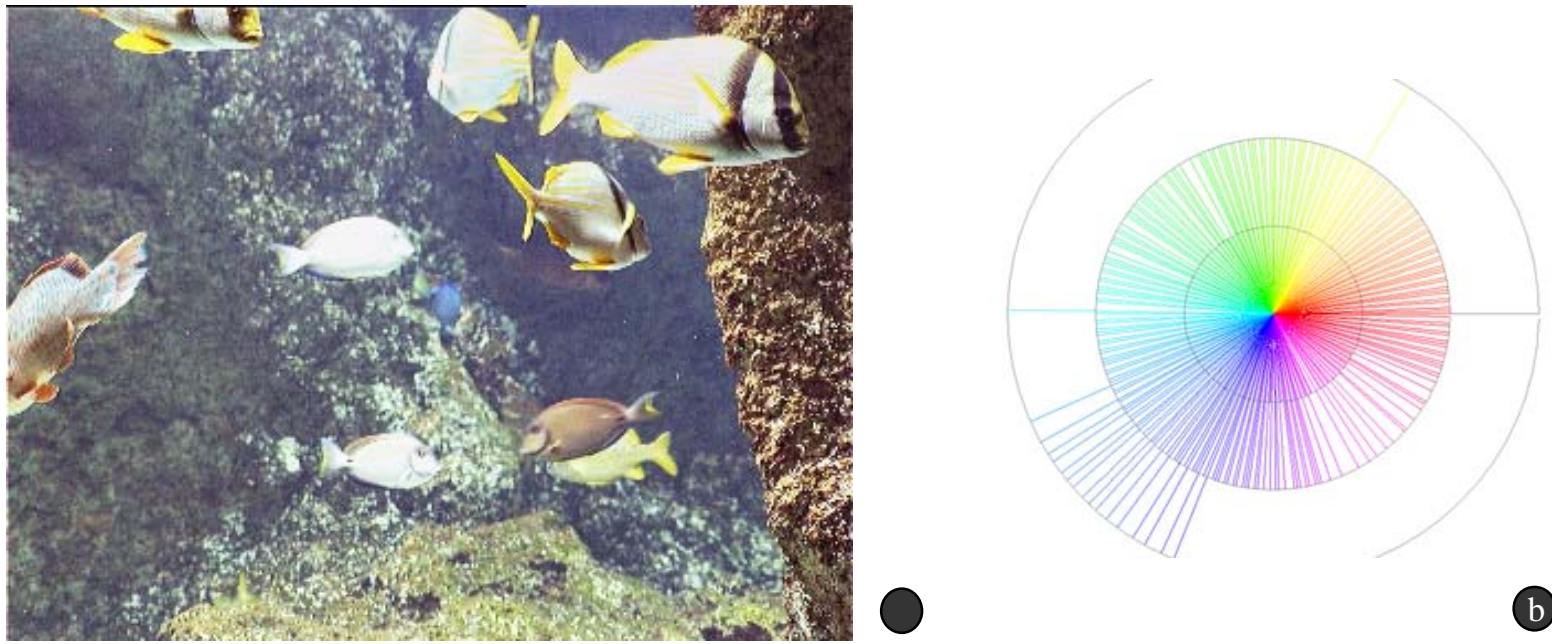

Fig. 8 : Image of fig. 3 after ACE without « keep original gay » feature (a) and its hue histogram showing its good chromatic diversity(b). 


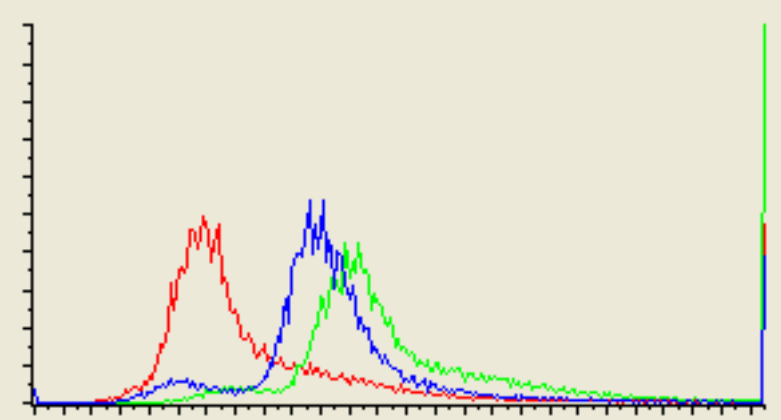

Fig. 9 : RGB histogram of fig. 3, the dynamic range is poor and there are clipping problems.

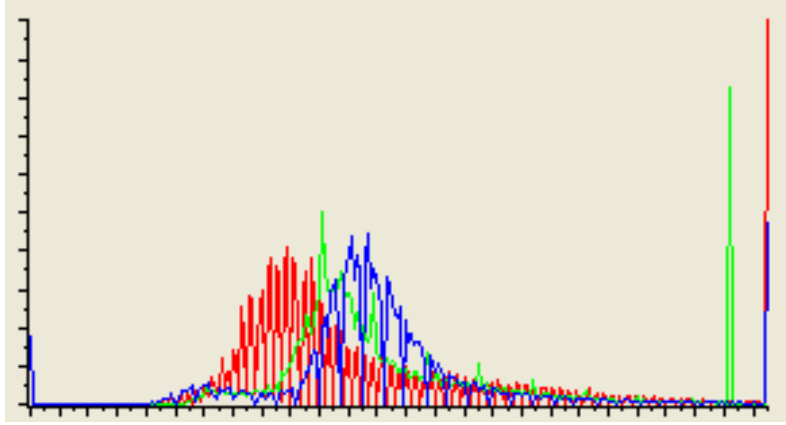

Fig. 11: RGB histogram of fig. 5 the dynamic range is still poor and there are clipping problems.

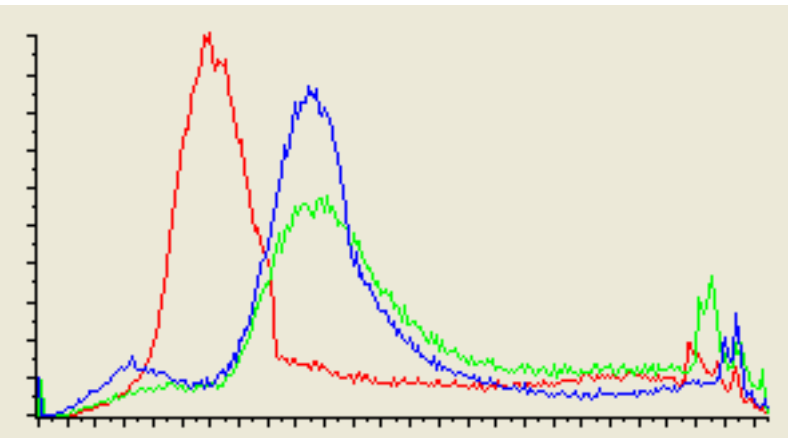

Fig. 13 : RGB histogram of fig. 7, it has a better dynamic range without equalization effects.

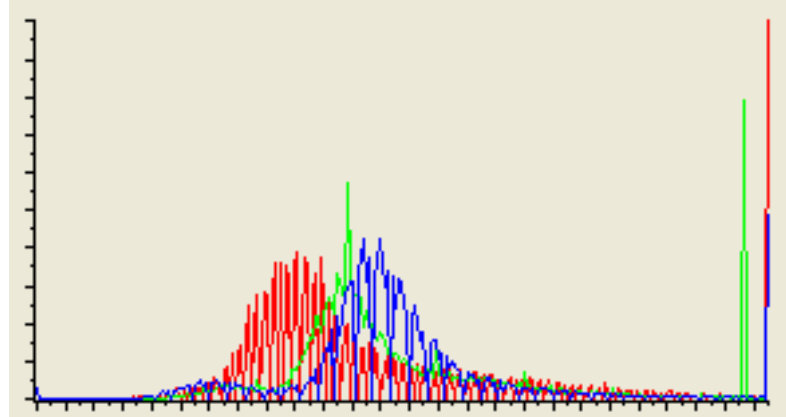

Fig. 10 : RGB histogram of fig. 4 the dynamic range is still poor and there are clipping problems.

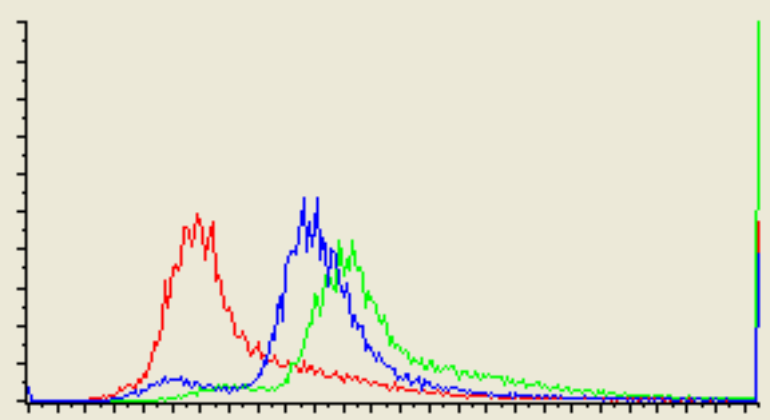

Fig. 12: RGB histogram of fig. 6, the histogram is similar to original one.

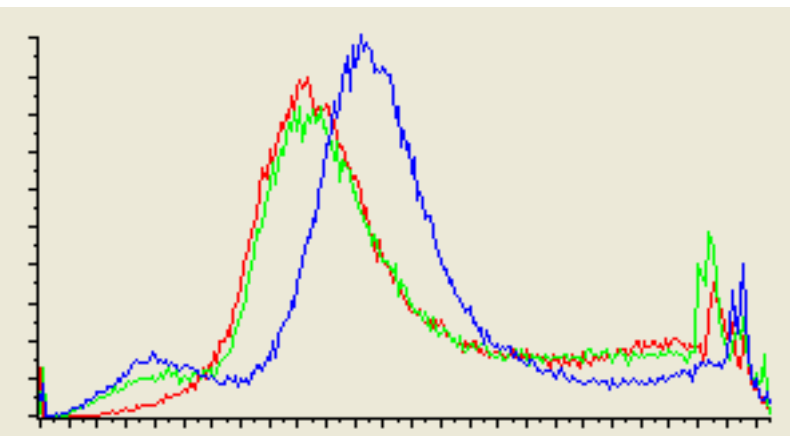

Fig. 14 : RGB histogram of fig. 8, it has a better dynamic range without equalization effects. 

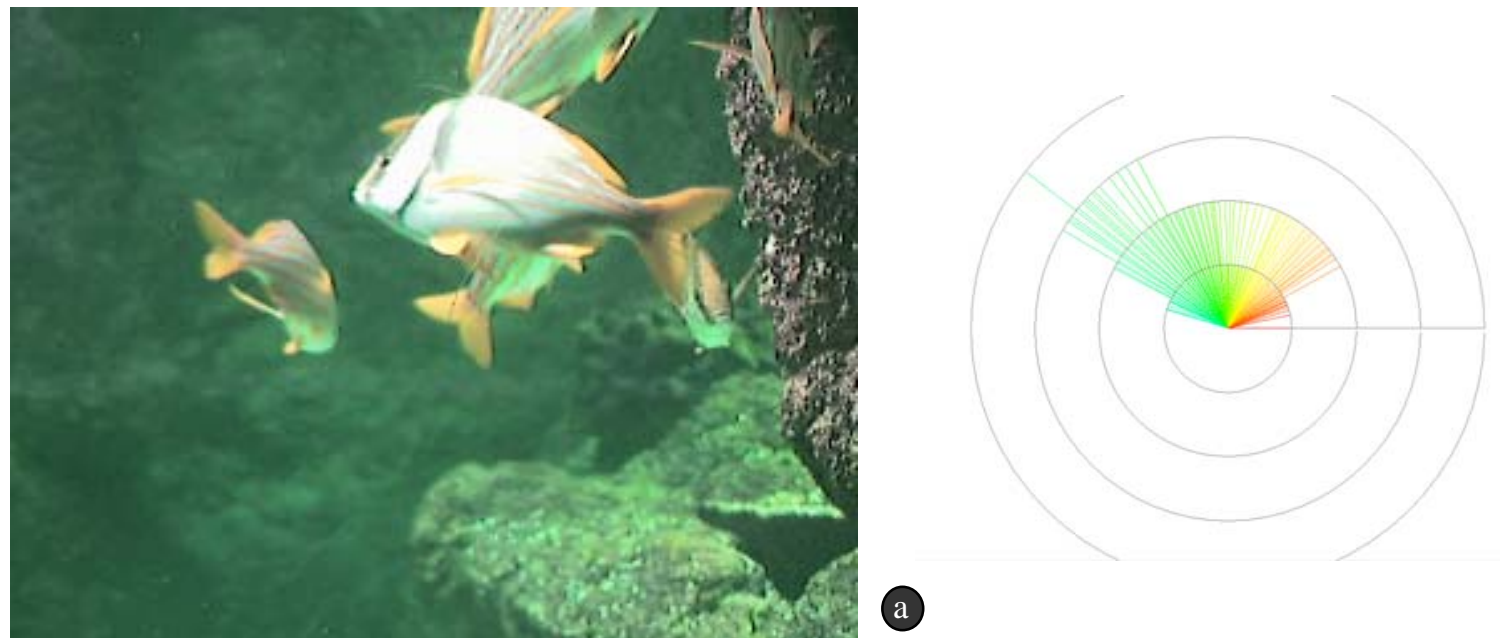

Fig. 15 : Original image with one kind of fish species (a) and its hue histogram (b).
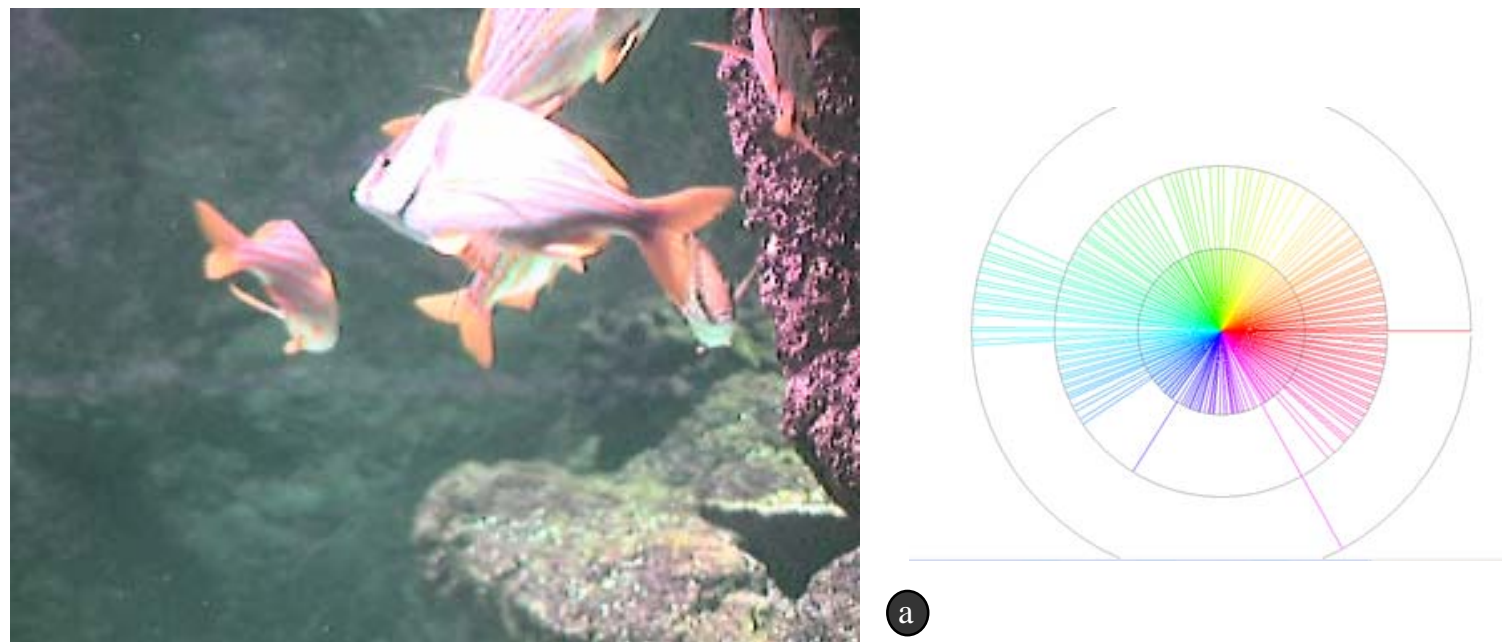

Fig. 16 : Image of fig. 15 after GW (a) and its hue histogram (b). Despite a certain chromatic diversity there is a strong reverse cast.
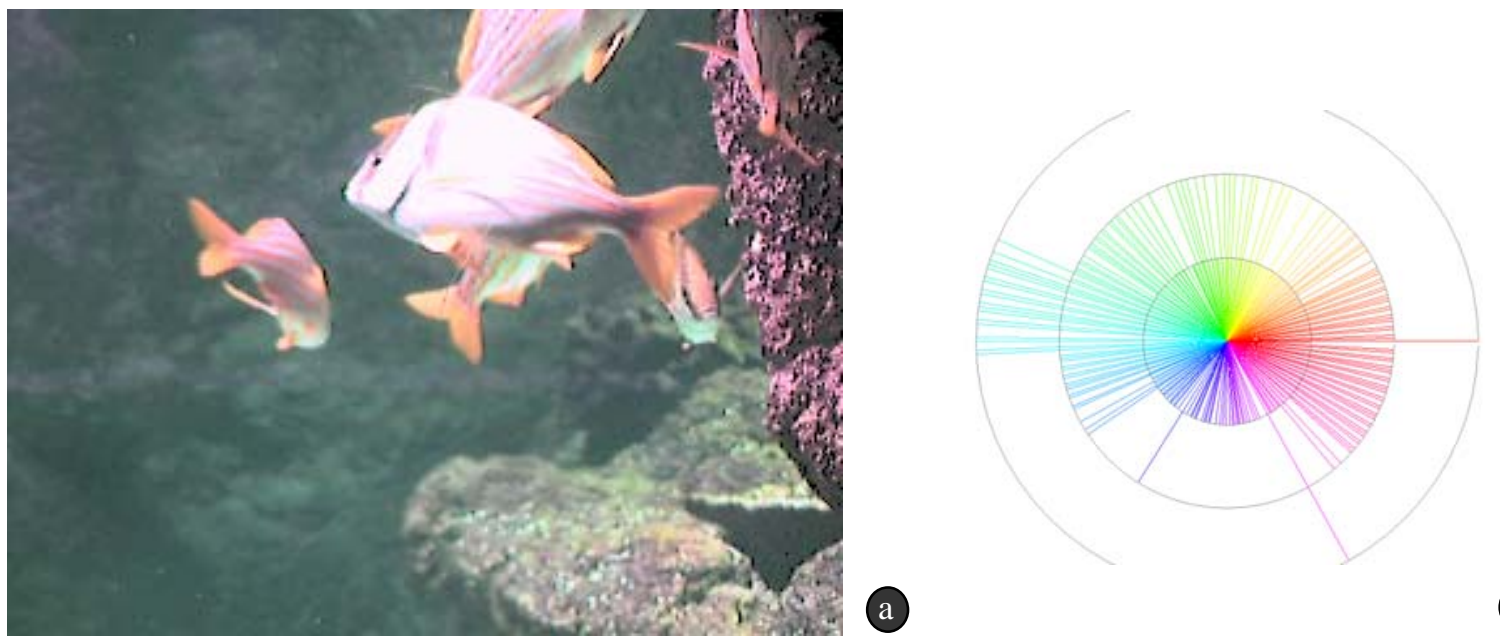

(b)

Fig. 17: Image of fig. 15 after GW/WP (a) and its hue histogram (b). Like the GW result (fig. 16) the image processed with GW/WP has a reverse color cast (magenta) 

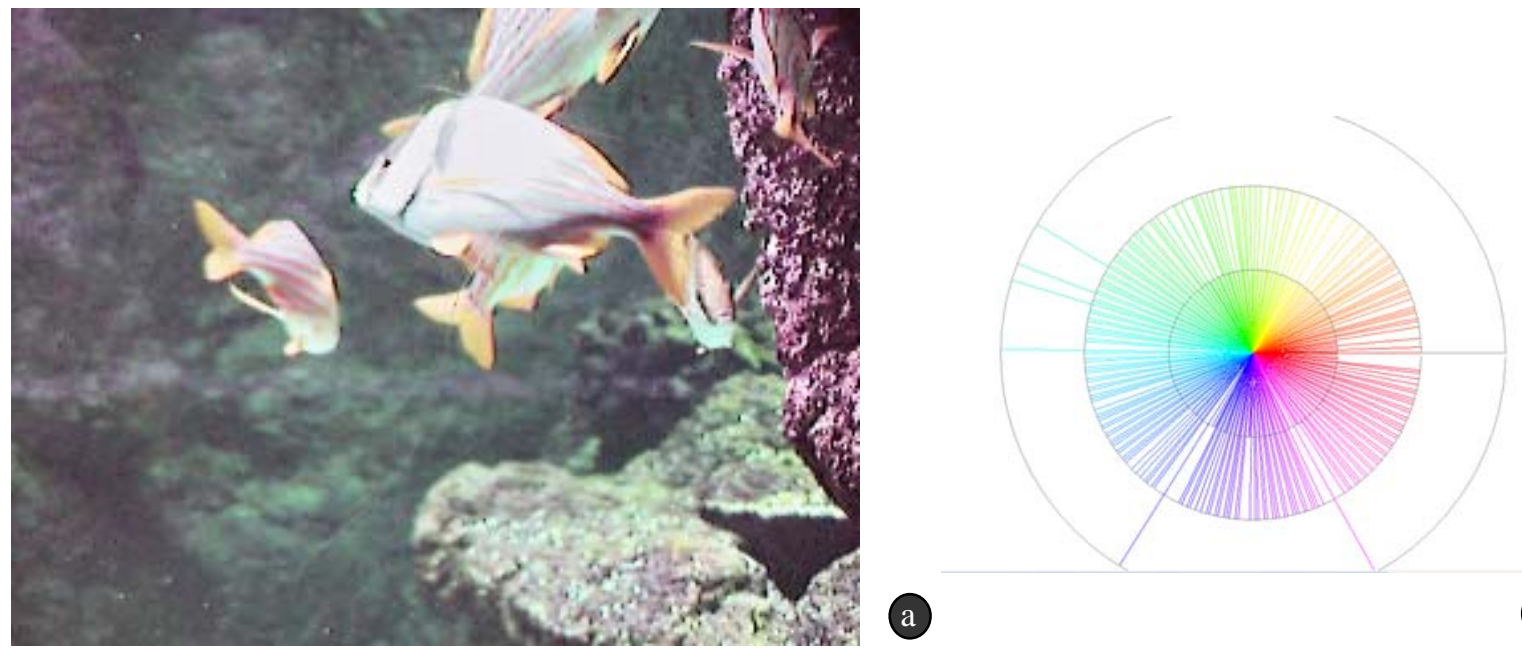

Fig. 18 : Image of fig. 15 after ACE (a) and its hue histogram (b). ACE keeps the real colors of the fish without introducing a reverse color cast, so it performs better during scenes with one variety of fish.
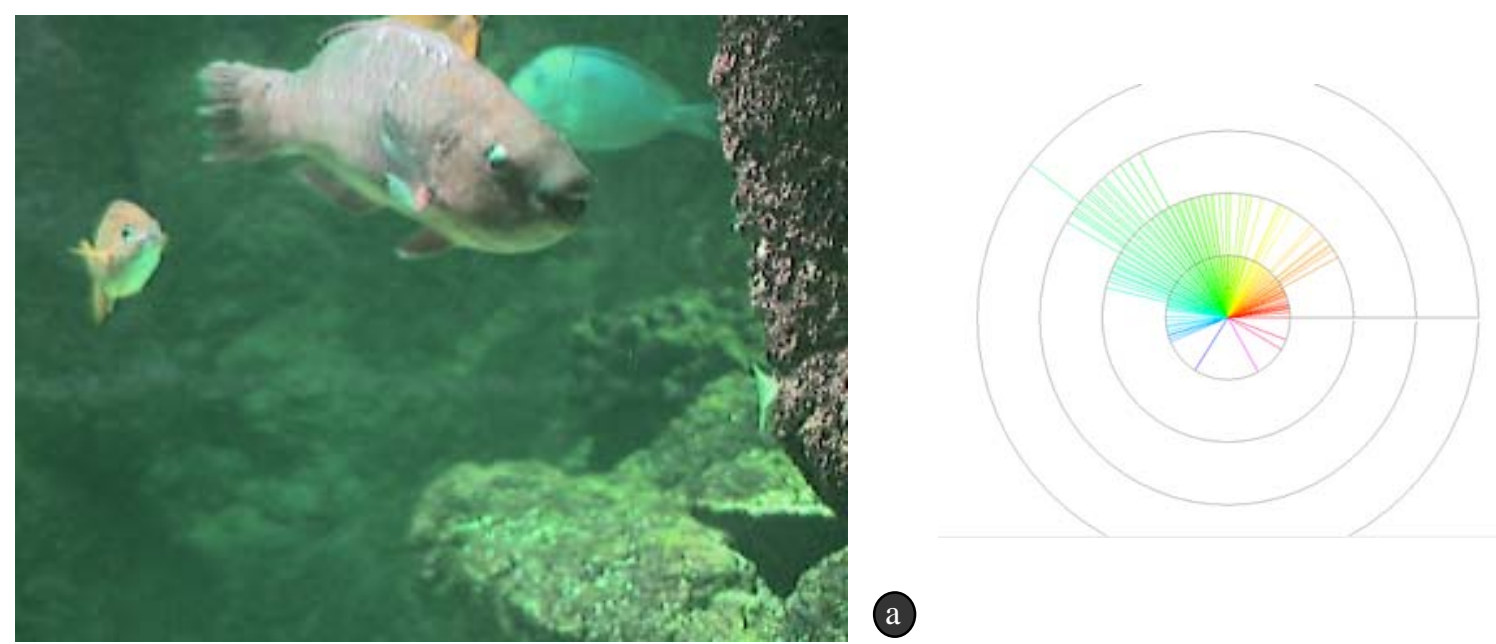

(a)

(b)

Fig. 19 : Original image, with a close-up on a fish (a) and its hue histogram (b).
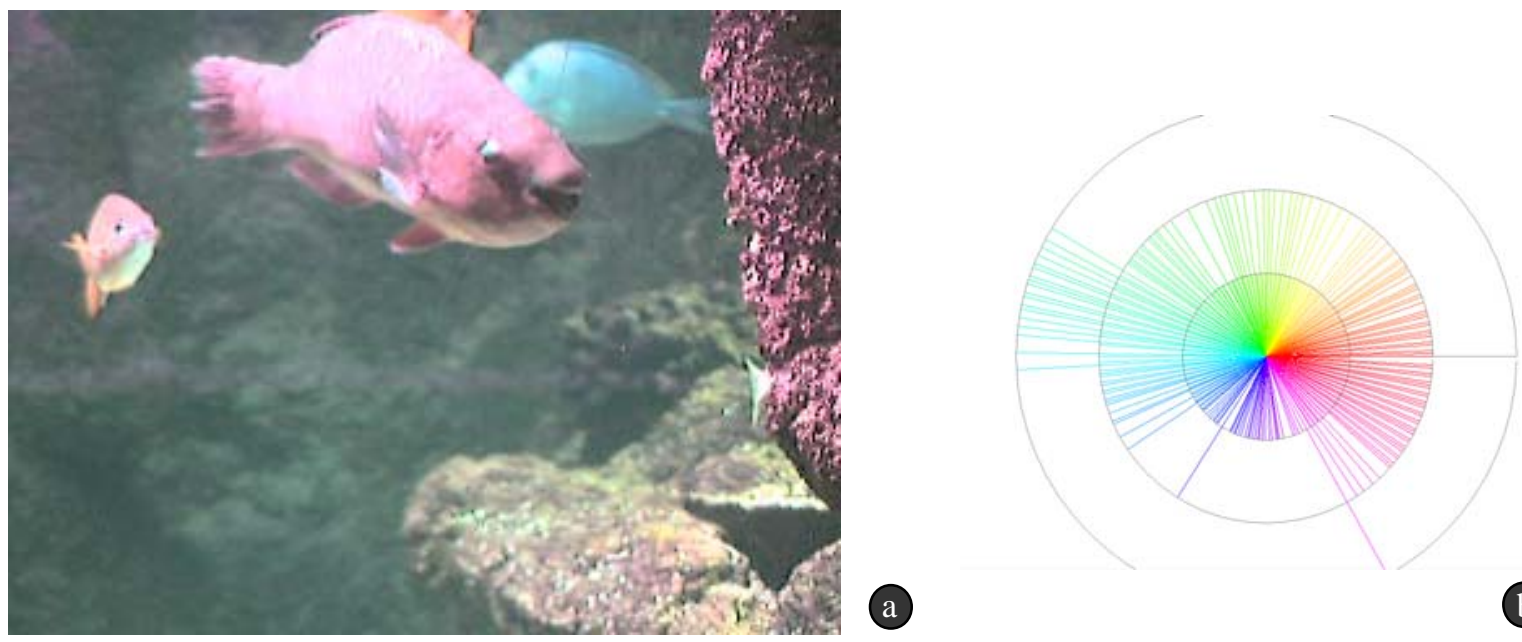

Fig. 20: Image of fig. 19 after GW (a) and its hue histogram (b). The fish color suffers from a reverse cast introduced by GW algorithm. 

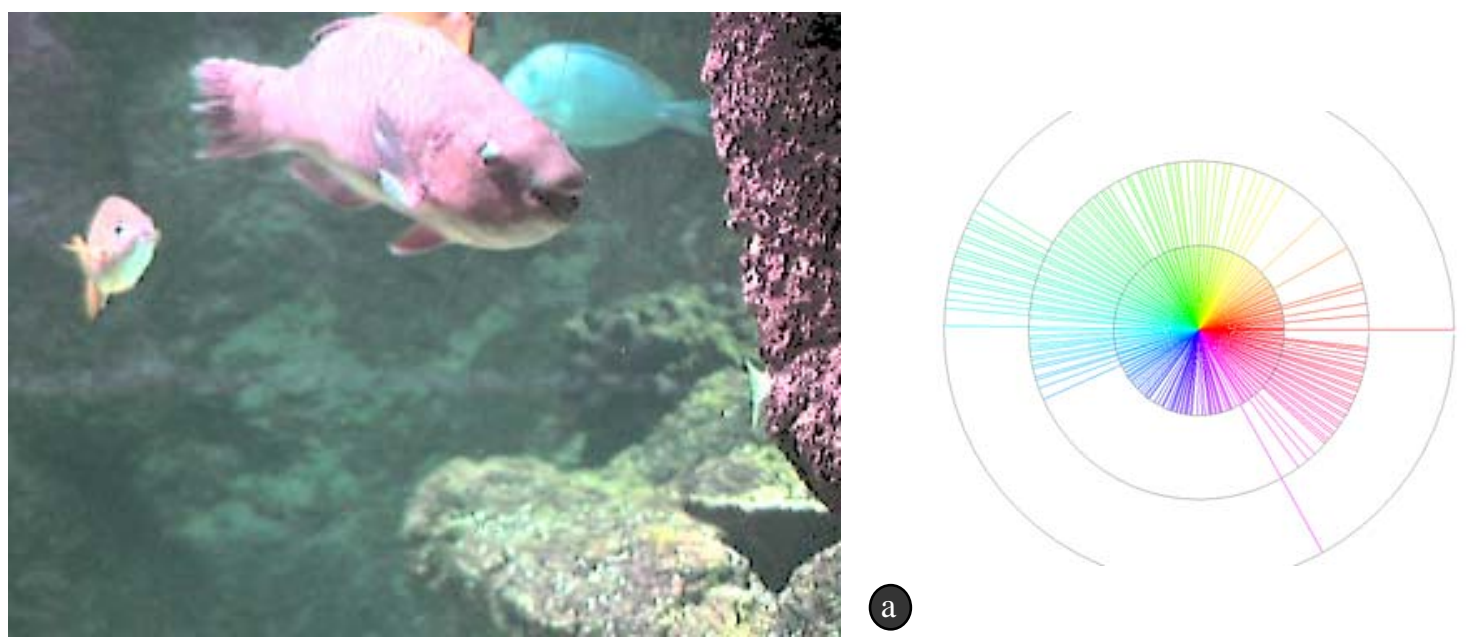

Fig. 21: Image of fig. 19 after GW/WP (a) and its hue histogram (b). GW/WP introduces less reverse cast than GW.
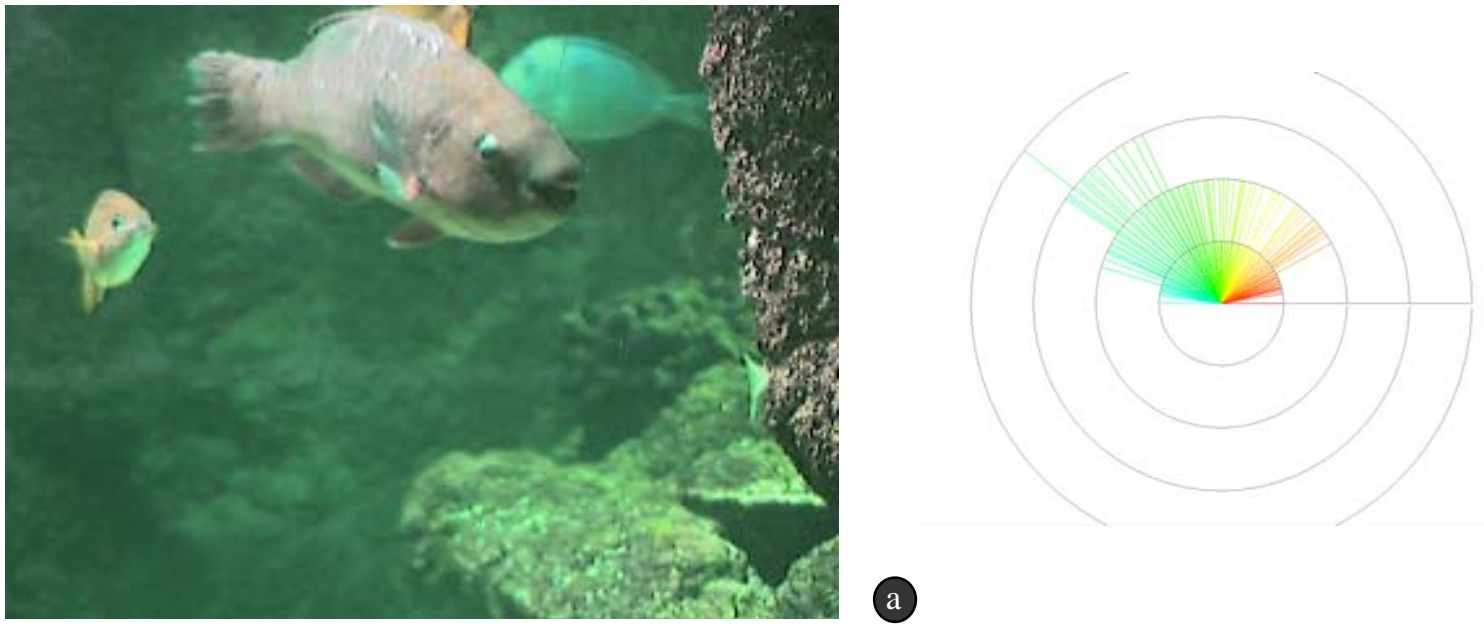

(b)

Fig. 22: Image of fig. 19 after WP (a) and its hue histogram (b). WP has nearly no effect on the image
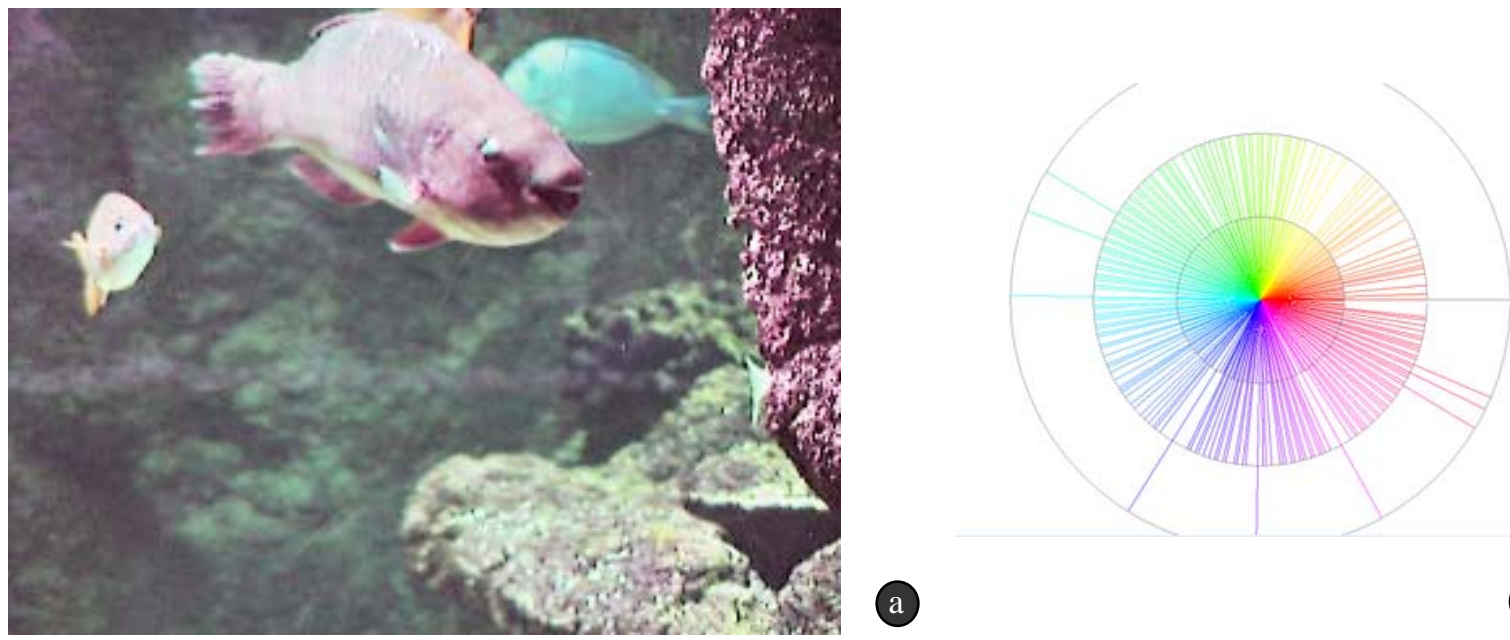

(b)

Fig. 23: Image of fig. 19 after ACE (a) and its hue histogram (b). ACE gives a better result with less magenta cast and better chromatic diversity 


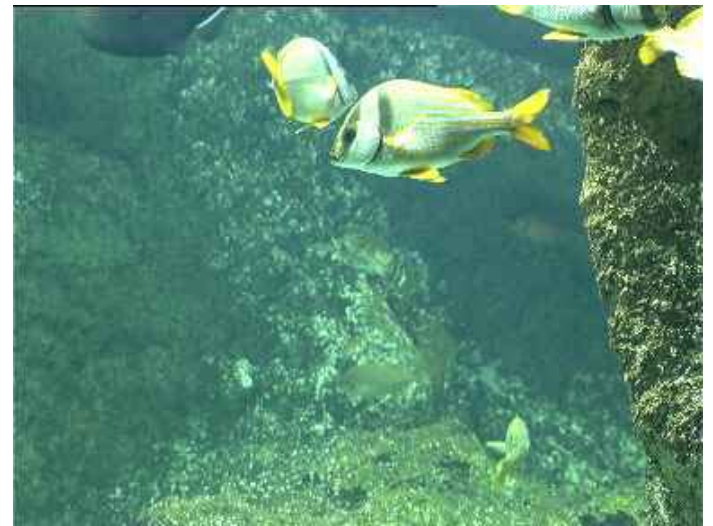

Fig.24.a : Original image

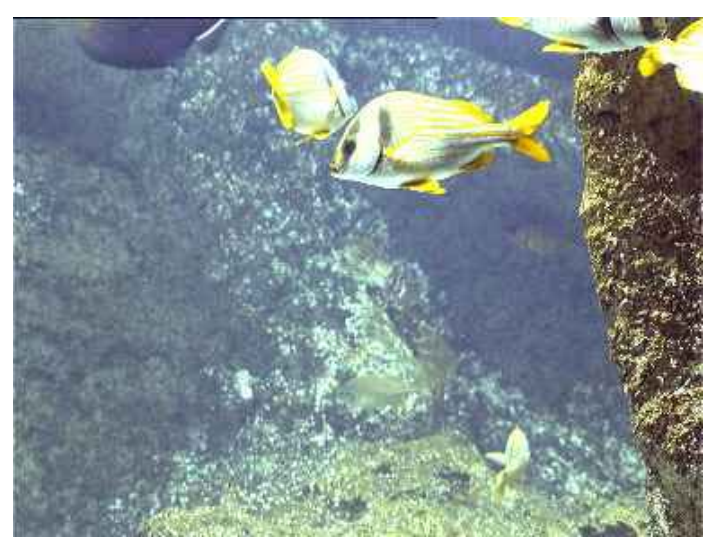

Fig. 25.a : The image of Fig. 24a after correction with ACE.

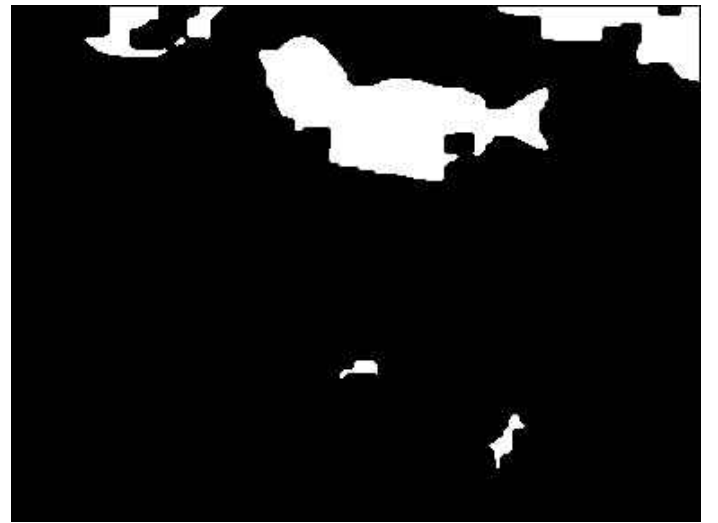

Fig. 24.b : Fish segmentation on the original image

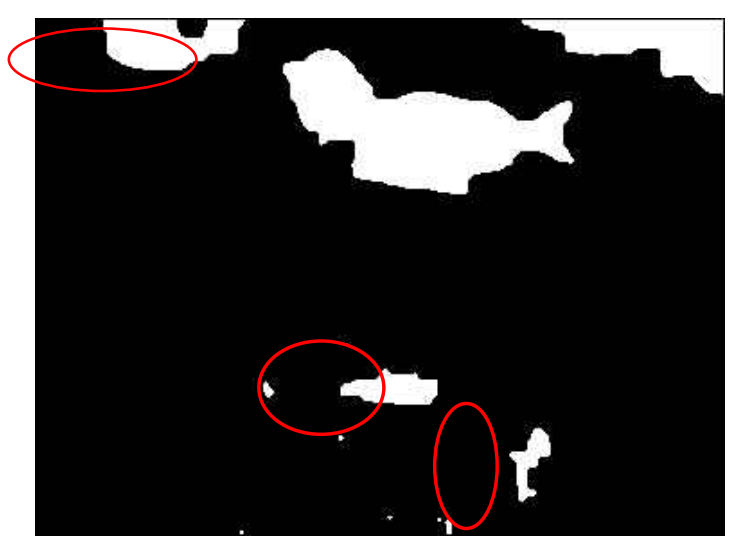

Fig. 25.b : Fish segmentation on the corrected image. We notice that the fish are better localized on the corrected image than on the original one. 